\title{
Dynamical structure factor of two-dimensional electrons over a helium film
}

\author{
Patricia Cristina Venturini, Nelson Studart, and José Pedro Rino \\ Departamento de Física, Universidade Federal de São Carlos, 13565-905, São Carlos, São Paulo - Brazil \\ E-mail: studart@df.ufscar.br
}

Received December 4, 2007

\begin{abstract}
The dynamical structure factor of a two-dimensional electrons trapped to a helium film deposited on a solid substrate is determined through molecular dynamics simulations for different film thicknesses and two substrates. The phases of the system varying from the classical Wigner crystal to strong and intermediate-correlated electron liquid as well the influence of the film thickness and substrate are analyzed in the numerical experiments.
\end{abstract}

PACS: $73.90+\mathbf{f}$ Other topics in electronic structure and electrical properties of surfaces, interfaces, thin films, and low-dimensional structures;

61.20.Ja Computer simulation of liquid structure.

Keywords: solid substrate, dynamical structure factor, helium film.

Since the pioneer work of Cole [1] and Shikin [2], surface electrons deposited on cryogenic materials, mainly liquid helium, have received considerable attention. For reviews about the subject see Refs. 3 and 4. Despite several reported studies of the dynamical properties of this interesting many-body electron two-dimensional (2D) system [5-14] the dynamical structure factor $S(\mathbf{q}, \omega)$, which is most relevant quantity to describe the collective behavior of the system, has been evaluated using simplistic approximations. Furthermore, $S(\mathbf{q}, \omega)$ is essential to study many-body effects in the transport properties such as the electron mobility [15]. Previous dynamical structure factors have been reported for the classical two-dimensional electron liquids $[16,17]$ in the intermediate and strong coupling regime, $2<\Gamma<70$, where the plasma parameter for the strictly $2 \mathrm{D}$ liquid $\Gamma=\left(e^{2} / T a\right)$, with the mean interparticle distance $a$ determined by $\pi a^{2} n=1, n$ is the electron density and the temperature $T$ is in energy units. However, the results were obtained from molecular dynamics simulations for a quite small system consisting of 81 particles. In this paper we consider a larger system with 784 particles and higher computational precision. Furthermore, the dynamical structure factor is evaluated to the more general system consisting of surface electrons floating over a helium film with thickness $d$ over a solid substrate with dielectric constant $\varepsilon$. By varying $d$, one can transform the interaction potential from a bare Coulomb potential to a dipolar one, making a system unique in this aspect. Several many-body properties of this electronic system have been analyzed in previous reports $[5,6,15,18,19]$.

The electrostatic potential energy is written as [5]

$$
U_{e e}=\frac{e^{2}}{2} \sum_{i, j=1}^{N}\left(\frac{1}{r_{i j}}-\frac{\delta}{\left[r_{i j}^{2}+(2 d)^{2}\right]^{1 / 2}}\right),
$$

where $N$ is the number of electrons, $r_{i j}=\left|\mathbf{r}_{i}-\mathbf{r}_{j}\right|$ (position vector in the $x y$ plane), and $\delta=(\varepsilon-1) /(\varepsilon+1)$. The helium dielectric constant is assumed to be one. In order to have charge neutrality, a uniform positive background is considered. For this system the plasma parameter can be defined as [8]

$$
\Gamma=\frac{e^{2}}{T a}\left[1-\delta\left(1+\frac{4 d^{2}}{a^{2}}\right)^{-1 / 2}\right] .
$$

The dynamical structure factor $S(\mathbf{q}, \omega)$ is defined by

$$
S(\mathbf{q}, \omega)=\frac{1}{2 \pi} \int_{-\infty}^{+\infty} F(\mathbf{q}, t) \mathrm{e}^{-i \omega t} d t,
$$

with the intermediate scattering function given by

$$
F(\mathbf{q}, t)=\frac{1}{N}\langle\rho(\mathbf{q}, t) \rho(-\mathbf{q}, 0)\rangle,
$$

where 


$$
\rho(\mathbf{q}, t)=\sum_{k=1}^{N} \exp \left[i \mathbf{q} \cdot \mathbf{r}_{k}(t)\right]
$$

is the density fluctuation function [20]. The temporal average $<>$ is taken over a finite time $\tau$ defined as the total time of the MD simulation run. By Fourier transforming the Eq. (5) the dynamical structure factor can be rewritten as

$$
S(\mathbf{q}, \omega)=\frac{1}{2 \pi N \tau}|\rho(\mathbf{q}, \omega)|^{2} .
$$

Molecular dynamics simulations were performed for a system consisting of 784 electrons and density $n=$ $=1.477 \cdot 10^{8} \mathrm{~cm}^{-2}$. The long range electron-electron interaction was handled using the Ewald method and the classical equations of motion integrated using the velocity Verlet algorithm with a time step of $0.5 \mathrm{ps}$, which conserve the total energy of the system better than one part in $10^{4}$ over the entire simulation. Periodic boundary conditions are imposed. For all studied cases, the system is allowed to be thermalized for at least 10.000 time steps, with averages taken over additional 10.000 time steps. The simulation starts with an electron lattice and successive heating and thermalization procedures lead to distinct phases at different temperatures.

The dynamical structure factor was calculated for wave vectors in the range $0.12<q a<4.7$, and frequencies in the range $0<\omega / \omega_{p}\left[(\pi n)^{1 / 2}\right]<2$, where $\omega_{p}(q)=\left(2 \pi n e^{2} q / m\right)^{1 / 2}$ is the plasma frequency for the strictly $2 \mathrm{D}$ electron sheet $[16,17]$. The smallest wave number considered is due to the finite size of the system.

Figure 1 displays the map of the dynamical structure factor $S(q, \omega)$ for surface electrons on bulk helium (very large $d$ ) in the very strong coupling $\Gamma=360$.

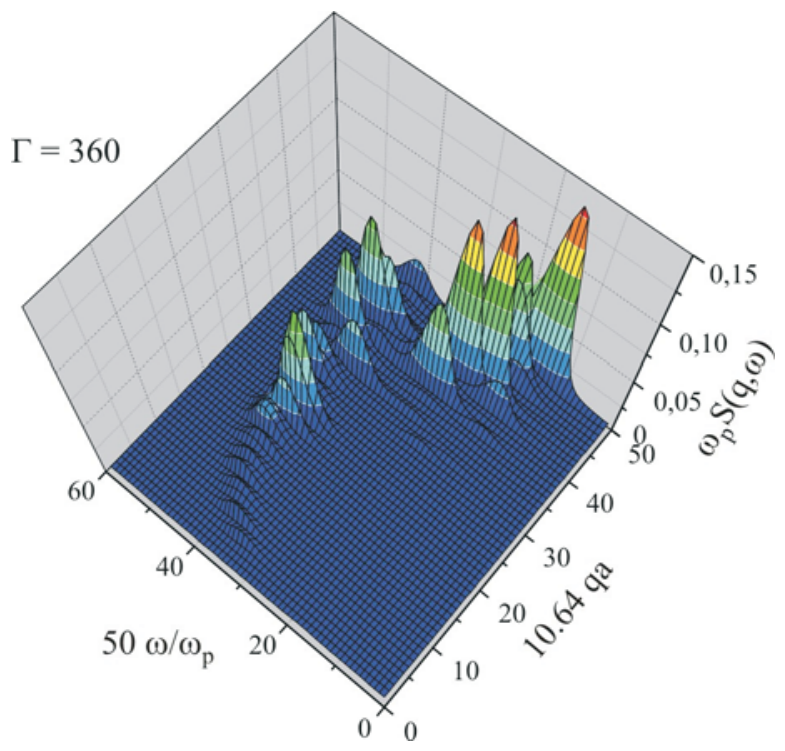

Fig. 1. $S(q, \omega)$ for a $2 \mathrm{D}$-electron system over bulk helium for $\Gamma=360$.

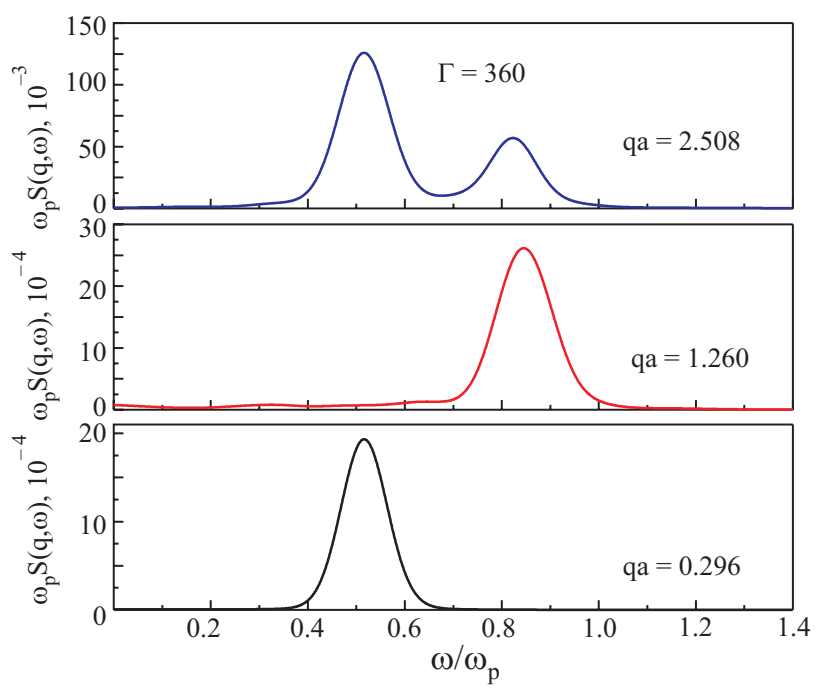

Fig. 2. $S(q, \omega)$ for a $2 \mathrm{D}$-electron system over bulk helium for $\Gamma=360$ and three wave vectors.

In this case, the system is in the solid phase, the classical Wigner crystal, and the collective mode at low frequencies can be clearly observed. In Fig. 2, we depict $S(q, \omega)$ for selected wave numbers. We note that by increasing $q$, the plasmon mode shifts towards $\omega_{p}\left[(\pi n)^{1 / 2}\right]$. With further increasing of $q$ this mode still shifts to lower values of frequency and a new peak closer to $\omega_{p}\left[(\pi n)^{1 / 2}\right]$ appears. This peak corresponds to the transversal shear mode which has been reported previously [5].

Figures 3 and 4 are plots of $S(q, \omega)$ for a lower value of $(\Gamma=90)$. A single collective mode at low $q$ is clearly distinguished. Again by increasing $q$, the mode peak moves to frequencies closer to $\omega_{p}\left[(\pi n)^{1 / 2}\right]$. One can see that at still larger values of $q$ the collective mode merges into a

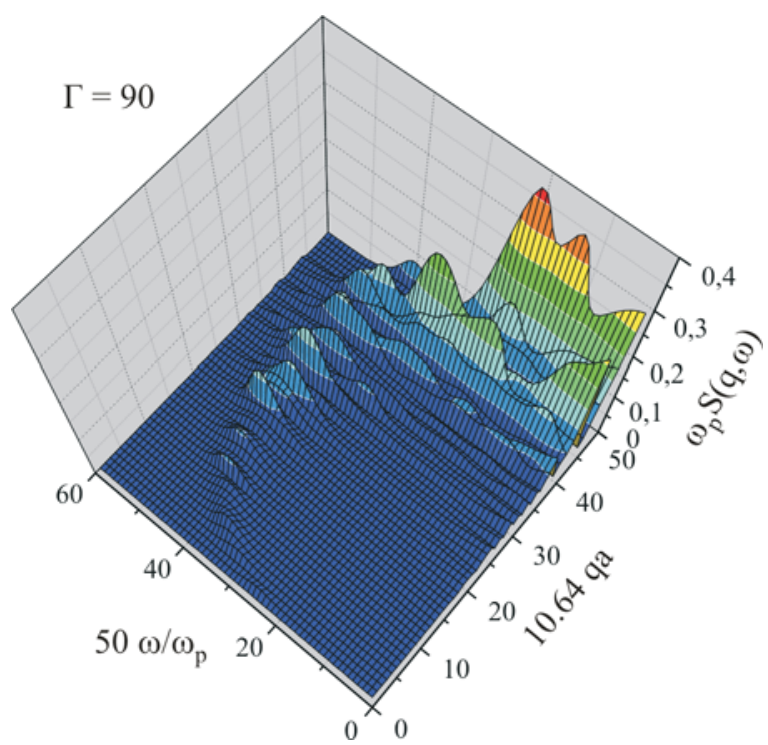

Fig. 3. Same as Fig. 1 for $\Gamma=90$. 


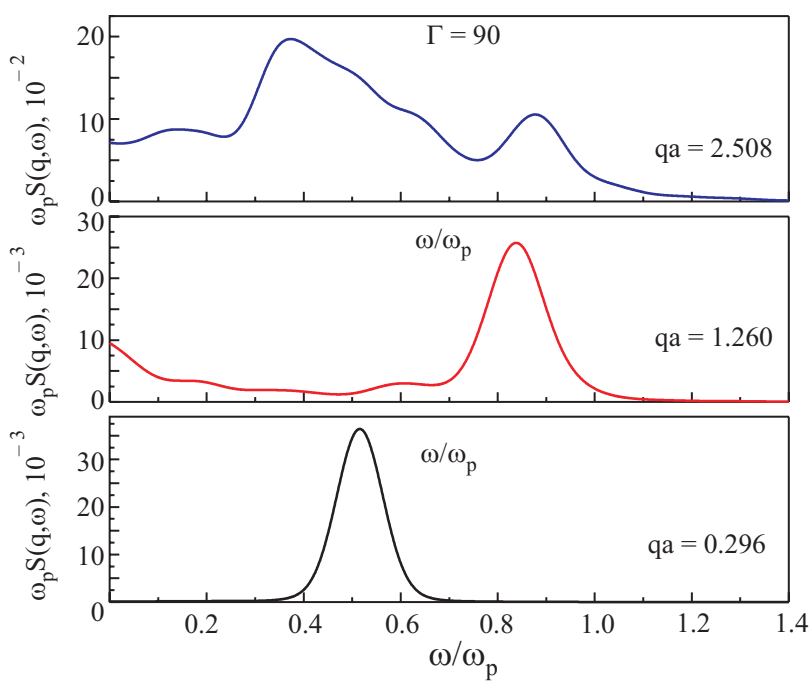

Fig. 4. Same as Fig. 2 for $\Gamma=90$.

continuum of excitations. The Rayleigh peaks are also presented for low $q$, which evidences the diffusive nature of the system characteristic of one particle excitations.

Now, we show in Fig. 5 the results of the dynamical structure factor for surface electrons on a thin film with $d=100 \AA$ A over a vitreous substrate, $\delta=0.75$, for two values of $\Gamma$. In this case, the collective mode appears at smaller values of frequency for the same wave vector when compared with the bulk spectrum.

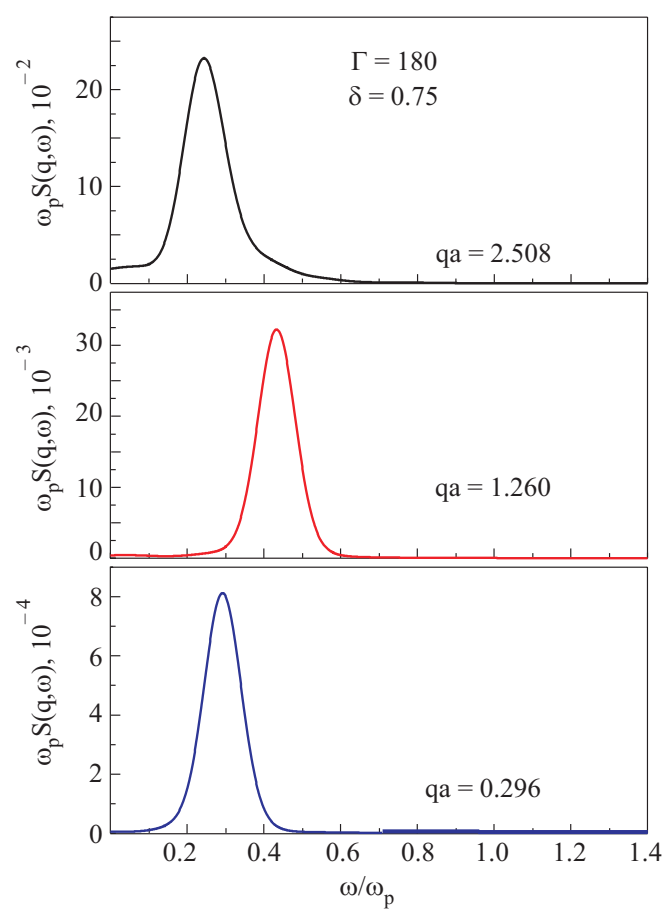

Essential differences are found when one considers a metal substrate. It has been shown [8] that the melting temperature, for a given film thickness, decreases with increasing the value of the dielectric constant, and the critical temperature reaches a minimum for a metal substrate $(\varepsilon=\infty)$. Figures 6 and 7 display the simulation results for $S(q, \omega)$ in the case of a film with thicknesses $d=100$ and $250 \AA$ A above a metal substrate $(\delta=1)$ for two different values of the plasma parameter. For the smallest wave vector, $q a=0.296$, the peak in $S(q, \omega)$ associated with the plasmon mode appears at frequency much smaller than $\omega_{p}\left[(\pi n)^{1 / 2}\right]$. We observe also that the plasmon peak decreases monotonically as the wave vector increases.

We have found that for a given film substrate, the plasmon mode shifts to higher values of frequency when the film thickness is increased. The dispersion relation has a linear dependence with $q, \omega \propto q$, as it is shown in the Fig. 8.

In summary, molecular dynamics simulations were performed in order to determine the dynamical structure factor for the two-dimensional electrons above a helium film deposited over a solid substrate. Results for different substrate and film thickness were obtained. These results are quite useful once it allow us to incorporate in a more accurate way many-body effects on the transport properties of the electrons following the method of the force-balance equation in which an effective mobility is

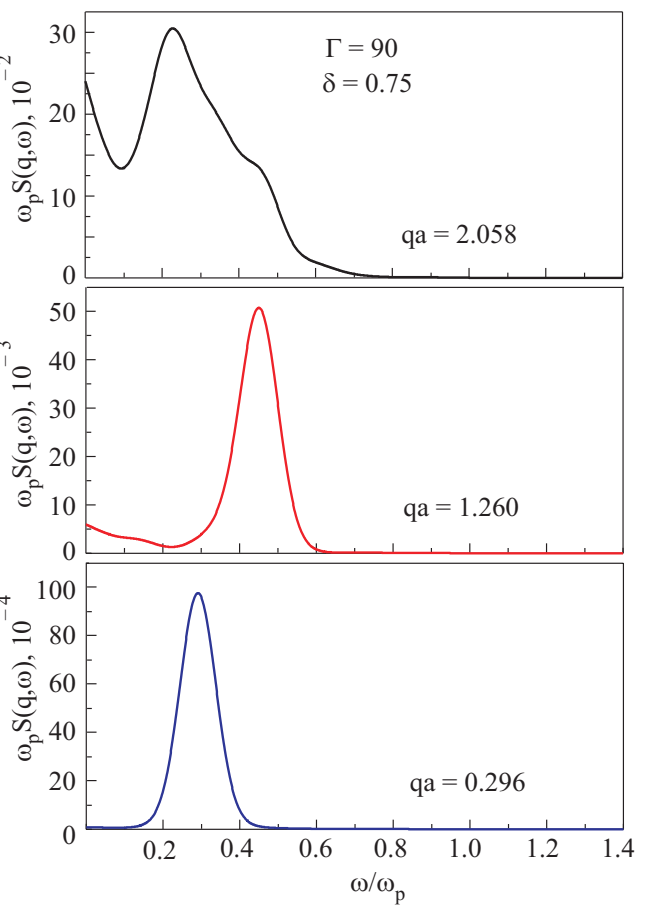

Fig. 5. $S(q, \omega)$ for a film thickness of $d=100 \AA$, a substrate with $\delta=0.75$ and $\Gamma=180$ (left) and 90 (right). 

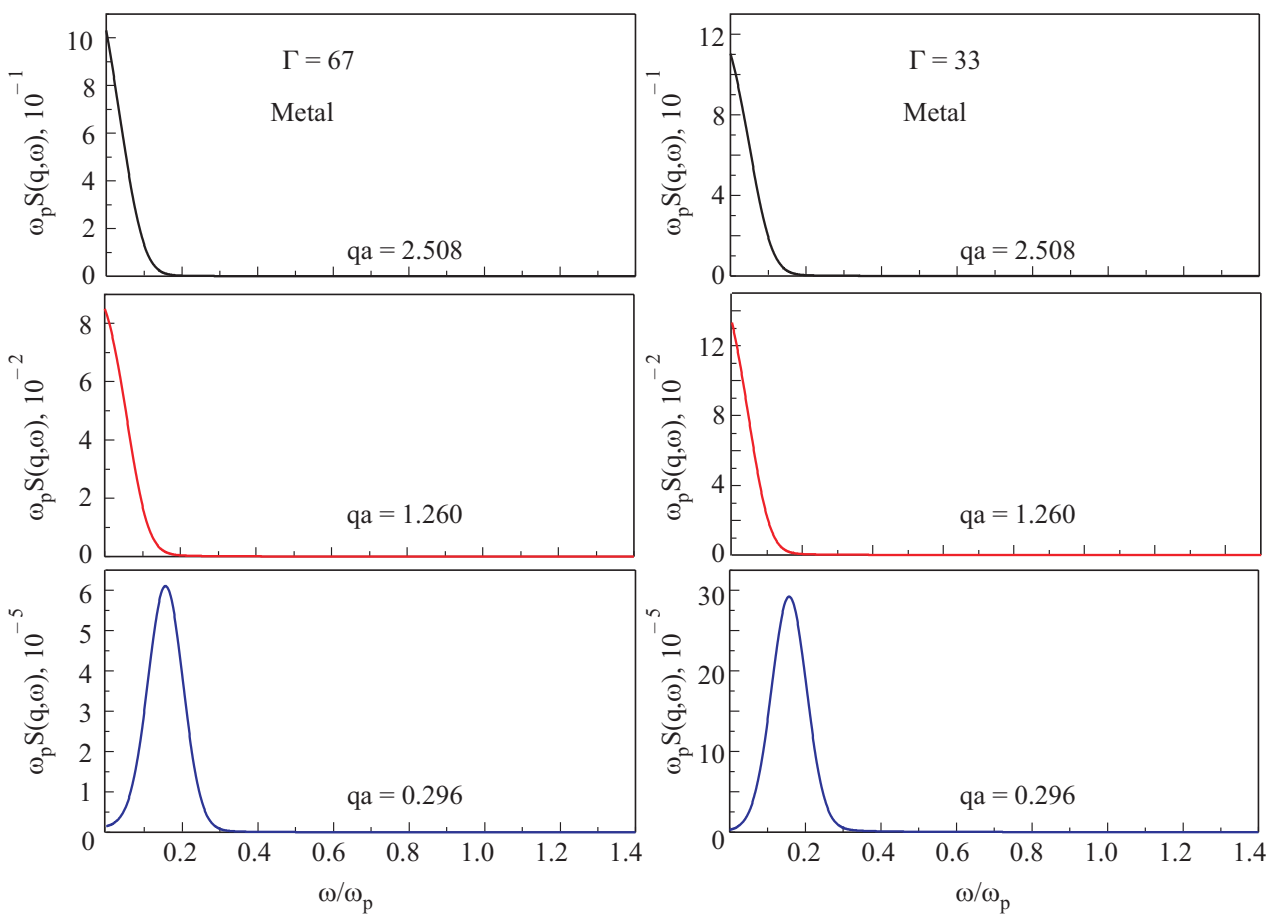

Fig. 6. Same as Fig. 5 for a metal substrate $(\delta=1)$ and $\Gamma=67$ (left) and 33 (right).
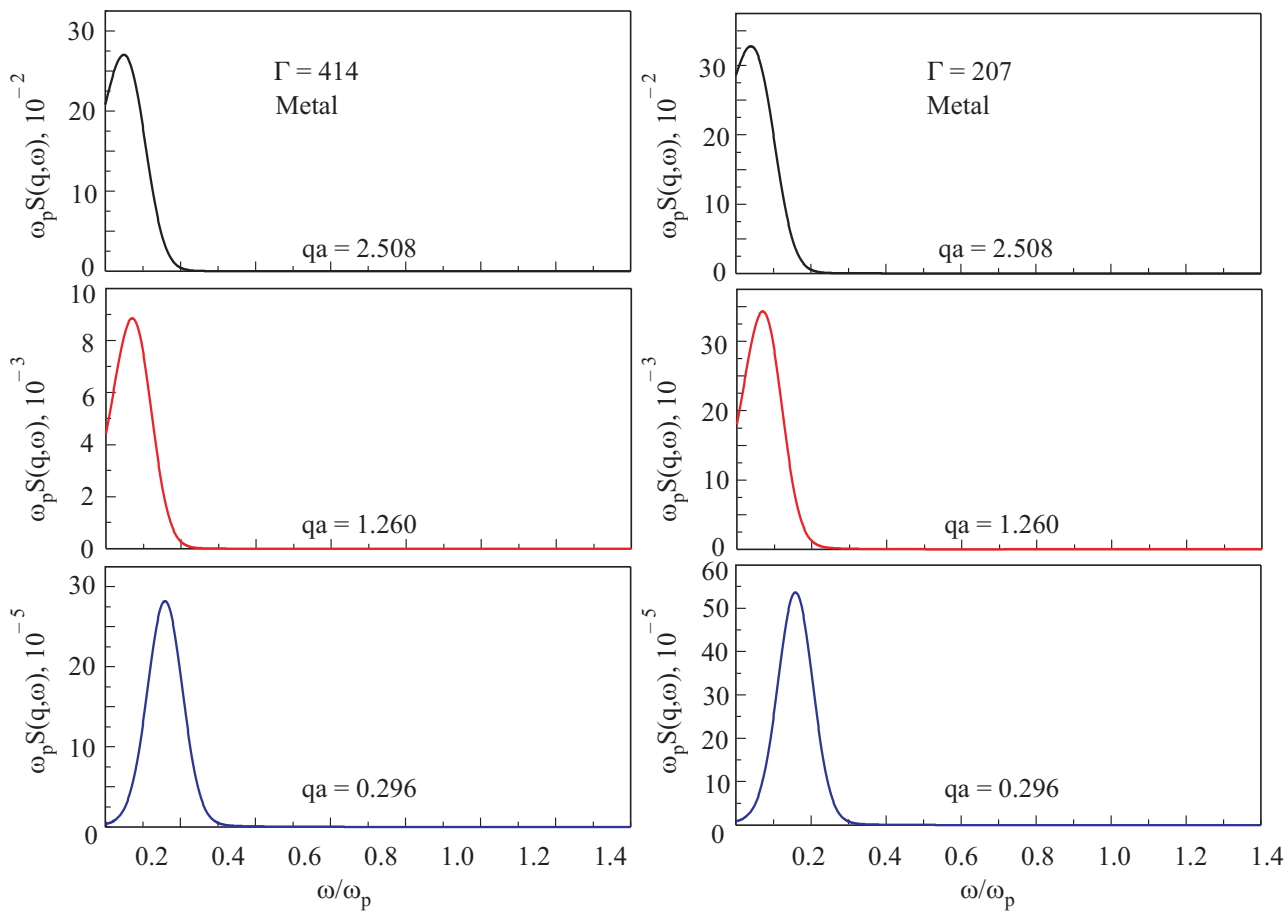

Fig. 7. Same as Fig. 6 for $d=250 \AA$ and $\Gamma=414$ (left) and $\Gamma=207$ (right). 


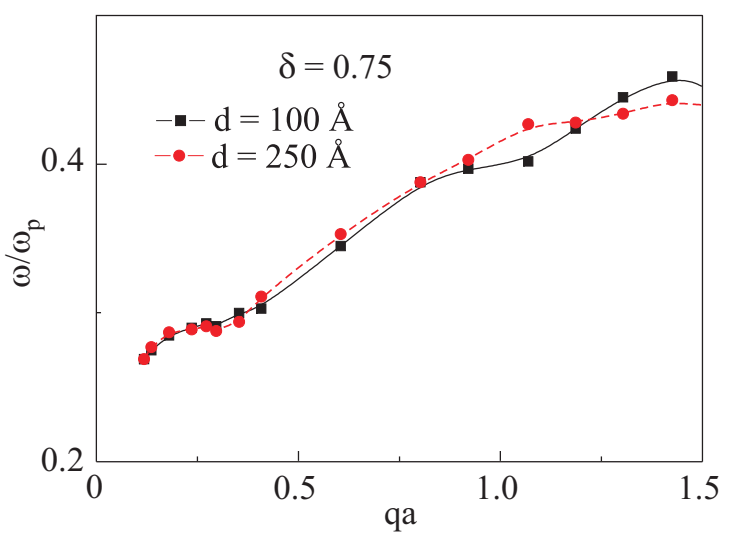

Fig. 8. Dispersion relation for electrons above a helium film adsorbed on vitreous substrate for $\Gamma=90$.

obtained in terms of the dynamical structure factor of the nondegenerate electron liquid [15].

The work was partially supported by FAPESP and $\mathrm{CNPq}$

1. M.W. Cole and M.H. Cohen, Phys. Rev. Lett. 23, 1238 (1969); M.W. Cole, Phys. Rev. B2, 4239 (1970).

2. V.B. Shikin, Zh. Eksp. Teor. Fiz. 58, 1748 (1970) [Sov. Phys. JETP 31, 936 (1970)].

3. Two-Dimensional Electron Systems on Helium and other Cryogenic Substrates, E.Y. Andrei (ed.), Kluver Academic, Dordrecht (1997).

4. Y.P. Monarkha and K. Kono, Two-Dimensional Coulomb Liquids and Solids, Springer, Berlin (2004).
5. F.M. Peeters, Phys. Rev. B30, 159 (1984).

6. J.P. Rino, N. Studart, and O. Hipolito, Phys. Rev. B29, 2584 (1984)

7. G. Kalman, Y. Ren, and K.I. Golden, Contrib. Plasm. Phys. 33, 449 (1993).

8. L. Candido, J.P. Rino, and N. Studart, Phys. Rev. B54, 7046 (1996)

9. P.M. Platzman and N. Tzoar, Phys. Rev. B13, 3197 (1976).

10. Y.P. Monarkha and V.B. Shikin, Zh. Eksp. Teor. Fiz. 68, 1423 (1975)

11. Y.P. Monarkha and S.S. Sokolov, Fiz. Nizk. Temp. 7, 45 (1981) [Sov. Low Temp. Phys. 7, 22 (1981)].

12. Y.P. Monarkha and V.B. Shikin, Fiz. Nizk. Temp. 8, 563 (1982) [Sov. Low Temp. Phys. 8, 279 (1982)].

13. Y.P. Monarkha and S.S. Sokolov, Fiz. Nizk. Temp. 8, 1235 (1982) [Sov. Low Temp. Phys. 8, 625 (1982)].

14. Y.Z. Kovdrya and Y.P. Monarkha, Fiz. Nizk. Temp. 12, 1011 (1986) [Sov. Low Temp. Phys. 12, 571 (1986)].

15. D. Coimbra, S.S. Sokolov, J.P. Rino, and Nelson Studart., Phys. Rev. B74 (2006).

16. H. Totsuji and H. Kakeya, Phys. Rev. A22, 1220 (1980).

17. H. Totsuji and H. Kakeya, Phys. Lett. A73, 23 (1979).

18. S. Sokolov, N. Studart, and D. Coimbra, J. Low Temp. Phys. 138, 409 (2005).

19. S.S. Sokolov, J.P. Rino, and Nelson Studart, Phys. Rev. B55, 14473 (1997).

20. N.H. March and M.P. Tosi, Atomic Dynamics in Liquids, Wiley, New York (1976). 